- All respondents reported they were 'very satisfied or fairly satisfied' with the service.

A virtual HBV pathway can deliver safe, evidence-based and timely surveillance with a high degree of patient engagement and satisfaction.

\section{GLOBAL FELLOWS: AN INTERNATIONAL CONTRIBUTION TO THE WORKFORCE CRISIS IN RADIOLOGY}

Robin D Proctor. University Hospitals of Morecambe Bay NHS Foundation Trust

\subsection{6/leader-2019-FMLM.99}

Diagnostic imaging is central to almost all diagnostic processes, particularly cancer care. Capacity for diagnostic imaging tests is one of the major limiting factors in the modern NHS and this is critically limited by a lack of radiologists.

The UK has a sound subspecialty training structure in radiology but insufficient current workforce. Specialist Training places for radiologists, although over subscribed at ST1 with high completion rates, also do not provide sufficient supply to change the situation.

India is a country of c.1.3billion people and trains a huge number of general radiologists to a high standard but offers more limited opportunities for structured higher training within a sub specialty. Thus there is a great wish to 'earn, learn and return' - to develop a subspecialty and knowledge of the NHS.

This is the report of leading a successful pilot of recruiting and placing radiologists who can contribute to the workload in the NHS while up-skilling themselves and returning to India with greater subspecialty expertise. We also offer an early report of further progress: scaling up across England, working with Health Education England (HEE), the Royal College of Radiologists (RCR) and Apollo Radiology International.

By implementing this scheme more radiologists are being recruited, leading to better, quicker diagnosis: patients will have better access to more rapid and accurate diagnosis which can only improve satisfaction.

Acceptability to patients is a key component of the programme including aculturalisation of recruits to the NHS and other learning of NHS values making sure that patients are treated with respect and dignity in keeping with the NHS constitution.

We describe the challenges and successful strategies by which we have navigated them to deliver a truly cross-system project involving four organisations which could hardly be more different: an NHS trust, an arm's length body, a Royal College and a commercial company based in India.

\section{STRENGTHENING NEW PAEDIATRIC REGISTRARS' LEADERSHIP POTENTIAL}

Philippa Anna Stilwell. Northwick Park Hospital, London, UK

10.1136/leader-2019-FMLM.100

Background This project sought to improve paediatric trainees' confidence and leadership skills just before becoming core paediatric/neonatal registrars. This is notoriously a difficulty transition point in the paediatric training pathway and presents potential risk to patient safety.

Assessment of issue and analysis of its causes

Data about trainees' confidence and leadership capabilities at this transition point was collected via an electronic survey from prospective and previous registrars. The responses highlighted concerns about 1) knowledge in certain aspects of neonatal medicine and 2) leadership confidence at this crucial transition period.

Intervention In response to the feedback, a study day was carefully designed to address the critical learning needs. Each lecture/workshop was led by a different specialist consultant, senior paediatric trainee or advanced nurse practitioner. The sessions were interactive and included case-based discussions and situation judgement scenarios. Different leadership styles were explored throughout the day.

Measurement of improvement To assess the impact of the educational material delivered on the course, detailed feedback was collected in real-time from attendees $(n=60)$, throughout the course. Confidence before and after each session was assessed (on a scale of 1 to 4); confidence increased by an average of 1 point in every lecture delivered. 94\% of attendees felt that the talks would help to improve their clinical practice and leadership skills as new paediatric registrars. Overall, $100 \%$ of attendees rated the study day a ' 1 ' (out of 4 ) in terms of usefulness (where $1=$ extremely useful and 4 $=$ not useful).

Lessons learnt Careful planning and briefing of speakers enabled an effective study day to be delivered for prospective new registrars, increasing confidence in various aspects of their training at the crucial transition point between being an $\mathrm{SHO}$ and becoming a registrar.

\section{ESTABLISHING A TRAINEES NETWORK}

Nirojan Sivapathasundararajah, Claire Ingram. University Hospitals Coventry and Warwickshire, UK

\subsection{6/leader-2019-FMLM.10}

There were many training opportunities in leadership, management, finances and trust-wide projects which were under-utilized by doctors.

It was apparent that most of the Junior doctors were not aware of such opportunities, nor how to access them.

The inspiration to start this network began from my journey in exploring leadership. I was unaware of ample opportunities at our trust, and felt I needed to explore externally, I wasn't alone in this thinking.

By involving local leadership trainers, we explored ways to disseminate the information better.

We carried out a survey to assess the trainee's perception of the trust, as well as how much knowledge they have of the opportunities available:

- Only 54\% had any understanding of the learning opportunities available to them

- Only $26 \%$ felt it was easy to access relevant information from the trust

- Only 43\% felt supported in exploring new ideas and lead change

- Only 37\% felt enthusiastic about their future role in the NHS 
In addition, the comments from the trainees suggested being 'undervalued', 'forgotten about in hospital wide conversations relating to learning/change', and 'felt junior doctors are left to get on with it'.

\section{The Network features}

- Trainee leads from each speciality

- All network members in contact via email and WhatsApp

- Periodic newsletters with opportunities at the trust, circulated by the network members

- Extra training slots were generated to facilitate trainees in existing training workshops, such as in finance and management.

- Allows for cross speciality collaborations in projects, and also a direct access for trainees to the trust leaders.

- Trainees could share other opportunities available to them, such as committee reps.

The network is growing with increasing numbers of volunteers to take up the lead roles. We hope this is a step in the right direction in finding our future leaders.

\section{LEADING INNOVATION AND IMPROVEMENT}

Maria van Hove, Anya Gopfert. Junior Doctor, Previous National Medical Director's Clinical Fellow

\subsection{6/leader-2019-FMLM.102}

Low morale among junior doctors has been attributed to a loss of the 'firm' or feeling of belong to a team as well as an increasingly demanding job, reduced resources and significant rota gaps. Improved teamwork is also associated with improved clinical outcomes. It is essential that we invest in and prioritise teambuilding for clinical staff in order to improve their wellbeing and to improve outcomes for patients. We developed an interactive, educational team-building workshop delivered in the form of an escape room, as a two-hour session including an extensive debrief and time for reflection. The Aims of the session are a) for participants to build inter-personal relationships with their colleagues b) to gain an understanding of their own role in a team. We obtained feedback from participants immediately, and nine months after the session in the form of survey data and qualitative responses. Immediate and longterm feedback were extremely positive and suggested that the training had significant improved participants' knowledge and understanding of working in a team. All participants reported that they were able to get to know their colleagues better, and that the session was enjoyable. Participants reported an improved understanding of team dynamics, a deeper awareness of their own role within a team, an increased knowledge of common team challenges and reported feeling more prepared for managing team conflict and delegating tasks. Feedback from participants after nine months particularly emphasised how the training had helped them prioritise effective communication with their team. An escape room scenario provides a novel, fun and effective way to both build a team and to improve understanding of teamworking. Participants were able to learn in a fun, innovative way, and feedback indicated that the training was successful. This is an affordable, innovative intervention which can be used for improving morale among clinical teams.

\section{KEEP THE WIRES AWAY. A QI PROJECT TO REDUCE THE OVERUSE OF CARDIAC TELEMETRY BEDS IN CAUSEWAY HOSPITAL}

Maysa Salman. Causeway Hospital, UK

10.1136/leader-2019-FMLM.103

Introduction ECG monitoring has been and remains to be an integral part of patient care in hospital. Arrhythmia detection has been reported to affect the clinical management in around $3.4-12.7 \%$ of patients. The American Heart association in collaboration with the American College of Cardiology published a statement in 2004 addressing the use of non-intensive cardiac telemetries stratifying patients into 3 different categories: cardiac telemetry is indicated, may provide benefit, or is unlikely to provide benefit. 123 telemetry beds were requested for acute admissions in October, including 13 post lysis stroke patients costing more than 30000 GBP

Methods We have adapted the AHA recommendations and formulated a local telemetry protocol in Causeway. Copies of the adapted guidelines were incorporated in the medical admission booklets, and circulated among the nurses as well as the bed managers. Junior doctors were trained about the protocol. There were 2 parts for this project. Part 1 was aimed at reducing the number of inappropriate cardiac monitors being used, while part 2 was aimed at reducing the duration in which cardiac monitors were used for.

Results Among the admissions, around 30\% of referrals were inappropriate and not indicated. Looking into the financial aspect of it, each bed costs around 300 GBP per night, leading to a total of around 30000 GBP for the total admission per night. After launching the cardiac telemetry protocol, there were a total of 89 telemetry bed requests, of which only $3 \%$ were inappropriate or not indicated, and an overall reduction of $10 \%$ in all the admissions, and $26 \%$ reduction in the inappropriate referrals. There was an overall $28 \%$ reduction of cost, saving around 10000 GBP during the first month period.

Conclusion Telemetry monitoring requires both trained personnel and specialized equipment, thus making one of the most costly equipment in the hospital setting. Guidelines remain a tool to aid but not replace clinical judgment.

\section{INCREASING THE PROPORTION OF VERBAL HANDOVERS OF ACUTELY UNWELL PATIENTS AT TORBAY HOSPITAL}

${ }^{1,2}$ Esther Oluseyi Bamigboye*,${ }^{1}$ Yuanpei Zhang, ${ }^{1}$ Clarissa Grondin. ${ }^{1}$ Torbay Hospital, UK; ${ }^{2}$ Derriford Hospital, UK

\subsection{6/leader-2019-FMLM.104}

Introduction This quality improvement project, run by three Foundation year 1 (F1) doctors, occurred at Torbay Hospital, a district general hospital in Devon England. The aim was to make the process of F1 Acute Reviews of unwell patients during weekend safer and more efficient.

Method Over a 3-week period, the number of acute reviews F1s received via text or a phone call on Saturday and Sunday was collated to ascertain the percentage of verbal acute review handover. We surveyed and spoke to nurses and members of the Hospital-at-day-team to determine their feelings on the subject. 